CrossMark CrossMark
\& click for updates

Cite this: J. Mater. Chem. C, 2015, 3, 7394

Received 19th May 2015,

Accepted 16th June 2015

DOI: $10.1039 / c 5 t c 01425 \mathrm{e}$

www.rsc.org/MaterialsC

\title{
Entanglements in marginal solutions: a means of tuning pre-aggregation of conjugated polymers with positive implications for charge transport $\uparrow$
}

\author{
Hanlin $\mathrm{Hu},{ }^{a}$ Kui Zhao, ${ }^{a}$ Nikhil Fernandes, ${ }^{b}$ Pierre Boufflet, ${ }^{c}$ James H. Bannock, ${ }^{c}$ \\ Liyang Yu, ${ }^{a}$ John C. de Mello, ${ }^{c}$ Natalie Stingelin, ${ }^{d}$ Martin Heeney, ${ }^{c}$ \\ Emmanuel P. Giannelis ${ }^{e}$ and Aram Amassian*a
}

\begin{abstract}
The solution-processing of conjugated polymers, just like commodity polymers, is subject to solvent and molecular weight-dependent solubility, interactions and chain entanglements within the polymer, all of which can influence the crystallization and microstructure development in semi-crystalline polymers and consequently affect charge transport and optoelectronic properties. Disentanglement of polymer chains in marginal solvents was reported to work via ultrasonication, facilitating the formation of photophysically ordered polymer aggregates. In this contribution, we explore how a wide range of technologically relevant solvents and formulations commonly used in organic electronics influence chain entanglement and the aggregation behaviour of P3HT using a combination of rheological and spectrophotometric measurements. The specific viscosity of the solution offers an excellent indication of the degree of entanglements in the solution, which is found to be related to the solubility of P3HT in a given solvent. Moreover, deliberately disentangling the solution in the presence of solvophobic driving forces, leads consistently to formation of photophysically visible aggregates which is indicative of local and perhaps long range order in the solute. We show for a broad range of solvents and molecular weights that disentanglement ultimately leads to significant ordering of the polymer in the solid state and a commensurate increase in charge transport properties. In doing so we demonstrate a remarkable ability to tune the microstructure which has important implications for transport properties. We discuss its potential implications in the context of organic electronics and photovoltaics.
\end{abstract}

\section{Introduction}

Conjugated polymers have attracted great attention due to their extensive applications in electronic devices such as organic thin film transistors (OTFTs), organic photovoltaic cells (OPVs),

\footnotetext{
${ }^{a}$ Physical Sciences and Engineering Division, Solar and Photovoltaic Engineering Research Center, King Abdullah University of Science and Technology (KAUST), Thuwal, 23955-6900, Saudi Arabia. E-mail: aram.amassian@kaust.edu.sa

${ }^{b}$ Department of Applied Engineering and Physics, Cornell University, Ithaca, New York 14853, USA

${ }^{c}$ Department of Chemistry and Centre for Plastic Electronics, Imperial College London, London SW7 2AY, UK

${ }^{d}$ Department of Materials and Centre for Plastic Electronics, Imperial College London, London SW7 2AY, UK

${ }^{e}$ Department of Materials Science and Engineering, Cornell University, Ithaca, New York 14853, USA

$\dagger$ Electronic supplementary information (ESI) available: Viscosity of neat solvents and solutions with different states, Hansen solubility parameters of solvents and P3HT and other parameters of solvents, TEM images, Spano model analysis, UV-vis absorption spectroscopy, AFM images, normalized ED profile, transfer characteristics and output curves of P3HT TFT devices, parameters for tube model, GPC spectra. See DOI: 10.1039/c5tc01425e
}

organic light-emitting diodes (OLEDs) and so on. ${ }^{1-4}$ Conjugated polymers are semi-flexible or rigid backbone polymers with exciting electronic and optoelectronic properties due to the delocalization of $\pi$-electrons along and between the backbones. This is responsible for carrier transport within the polymer chain as well as among adjacent backbones. Conjugated polymer semiconductors have several advantages compared to their inorganic counterparts. These include lightweight, solution-processability and suitability for continuous and high throughput industrial manufacturing via roll-to-roll coating and printing routes..$^{5-8}$

Despite the great scientific and technological interests in this class of materials, much of the progress made in the polymer science field has focused on non-conjugated flexible polymers. Bueche developed a molecular theory in solution which accounted for the effects of chain entanglements on the viscoelastic properties. ${ }^{9}$ This approach is successfully used to this day, whereas theories for semi-flexible polymers are more complicated, since the behaviour of those polymer molecules is not only dominated by entropic effects but also by internal energy contributions. The competition of internal bending energy and entropy resulted in the concept of persistence length $l_{\mathrm{p}}$, which was treated as the 
average distance over which the polymer appears straight. De Gennes, ${ }^{10}$ Doi and Edwards, ${ }^{11}$ reduced the complicated phase space topology to a static environment in their seminal model for semi-flexible polymers: the tube model. This model explained how diffusion of a polymer chain occurs only along its contour rather than the other directions.

Achieving a deeper understanding of the relationship between the behavior of semi-flexible conjugated polymers in the solution state and their molecular and macroscale structure in the solid state is necessary and helpful to improving the performance of electronic and optoelectronic devices. Polymer chains are known to form entanglements in both solution and molten states, ${ }^{12,13}$ which can hinder molecular motion and impact the molecular assembly in the solid state. ${ }^{14-16}$ A higher molecular weight (weight-average molecular weight, $M_{\mathrm{w}}$ ) leads to a longer contour length, a decreased effective hydrodynamic volume, and therefore a higher chance to form binary hooking which result in more entanglements. In the solvated state, the polymer chains tend to assume the extended state with large hydrodynamic volume even for high $M_{\mathrm{w}}$. However, polymer chains prefer adopting more rigid properties in formulation conditions characterized by more solvophobic conditions. ${ }^{12}$ This results in the coexistence of polymer chain entanglements and aggregated precursor species. Ultrasonication has been successfully shown to promote aggregation of polymer chains in marginal solvents without damaging the polymer chain and is believed to provide the kinetic means for chain conformational changes in conditions where the solution approaches its solubility limit. ${ }^{12,17-19}$ This effect has been credited with promoting the formation of polymer thin films with enhanced backbone planarity, lamellar thickness and long range $\pi-\pi^{*}$ crystallinity in thin films. ${ }^{12,18-20}$ However, the ways in which changes in the formulation, such as solvent choice, molecular weight, and concentration, affect the entanglement and aggregation behaviour of a semi-flexible conjugated polymer solution remain ill understood. Furthermore, how these changes impact the microstructure and ultimately the transport properties of conjugated polymer semiconductors in the solid state is to date rather poorly understood.

In this study, we systematically investigate the solution state of the semi-flexible conjugated polymer P3HT in different technologically relevant solvents and related conditions (concentration and molecular weight) via rheometry and UV-Vis absorption measurements. At the limit of solubility in these different formulations we show that manipulating the entanglements allows to control the formation of pre-aggregates in solution which can subsequently be used to tune the local and long range order in P3HT thin films, with significant implications upon the transport properties of this semiconducting polymer. To do so, we have combined quantitative UV-Vis absorption in the solid state with transmission electron microscopy (TEM) and electron diffraction (ED), atomic force microscopy (AFM), and field effect mobility measurements in organic thin film transistors (OTFTs). The viscosity and UV-Vis analyses draw a clear picture of the solubility-entanglement-aggregation nexus and reveal the choice of solvent and $M_{\mathrm{W}}$ to be critical parameters. Faced with the combination of reduced solubility and increasing number of entanglements for the longer chain (higher $M_{\mathrm{w}}$ ) polymers, disentanglement is shown to reduce the viscosity of the solution and promote pre-aggregation of the polymer for a wide range of solvents used in electronic and photovoltaic applications. These results are explained on the basis of the tube model, whereby the disentanglement of the polymer chains in solution via ultrasonication facilitates the movement of polymer chains by alleviating the suppression and hindrance from surrounding polymer molecules, resulting in increased freedom of the polymer chains. These kinetic factors in solution are shown to promote the formation of polymer films with local and long range order exhibiting enhanced carrier transport.

\section{Materials and methods}

\subsection{Materials}

Regioregular P3HT $\left(M_{\mathrm{w}}=22,32 \mathrm{kDa}\right.$ and regioregularity, $\mathrm{RR}=$ 93.7\% and $94.7 \%$, Ossilla Co; $M_{\mathrm{w}}=68 \mathrm{kDa}$ and $\mathrm{RR}=98.5 \%$, Sigma-Aldrich Co; $M_{\mathrm{w}}=100,120$ and $180 \mathrm{kDa}$ were provided by the groups of Profs. John C. de Mello and Martin Heeney; the details of synthesis are reported elsewhere; ${ }^{21,22}$ all P3HT batches were subjected to gel permeation chromatography for determination of $M_{\mathrm{w}}$, as described in Section 2.7 and Fig. S1, ESI $\dagger$ ) and $\mathrm{CHCl}_{3}$ (99\% anhydrous; Sigma-Aldrich Co.), toluene (99.8\% anhydrous; Sigma-Aldrich Co.) chlorobenzene (99\% anhydrous; Sigma-Aldrich Co.), di-chlorobenzene (99\% anhydrous; SigmaAldrich Co.) were used without further purification.

\subsection{Solution preparation}

P3HT was dissolved in $\mathrm{CHCl}_{3}$, Tol, CB and oDCB at $60{ }^{\circ} \mathrm{C}$ for $10 \mathrm{~min}$ and then kept stirring in dark environment over one night at room temperature.

For the higher $M_{\mathrm{w}}(100 \mathrm{kDa}, 120 \mathrm{kDa}$ and $180 \mathrm{kDa})$, the solution just kept stirring in dark environment for 1 hour. The obtained solutions were placed in small glass vials and were treated with ultrasonication by using a Bransonic cleaner (model 1510E-DTH) with frequency of $42 \mathrm{kHz}$ at room temperature for $4 \mathrm{~min}$. In a previous study, we have proved that ultrasonication can result in disentanglement of polymer chains while maintaining the same polymer $M_{\mathrm{w}}$ as well as the chemical properties as demonstrated by GPC measurements performed before and after ultrasonication. ${ }^{19}$

\subsection{Device fabrication and characterization}

Highly doped n-type silicon wafers (100) with thermally evaporated $240 \mathrm{~nm} \mathrm{SiO}_{2}$ and gold electrodes were purchased from Fraunhofer Co. with a channel width $(W)$ of $10000 \mu \mathrm{m}$ and length $(L)$ of $2.5 \mu \mathrm{m}$. Initially the substrates were cleaned by rinsing with acetone, isopropanol, ethanol, and de-ionized (DI) water followed by Standard Clean 1 (RCA) ammonium hydroxide $\left(30 \% \mathrm{NH}_{4} \mathrm{OH}\right)$, hydrogen peroxide $\left(30 \% \mathrm{H}_{2} \mathrm{O}_{2}\right)$ and DI water (with $1: 1: 5$ ratio) for $15 \mathrm{~min}$ at $70{ }^{\circ} \mathrm{C}$. Finally all substrates were dried with $\mathrm{N}_{2}$ and heated at $100{ }^{\circ} \mathrm{C}$ for $10 \mathrm{~min}$. Different solutions (or solution state) of P3HT (with different $M_{\mathrm{w}}$ ) in different solvents were spin-coated (SPIN 150) onto substrates at a rate of $800 \mathrm{rpm}$ for $60 \mathrm{~s}$. All electrical measurements were 
performed with a Keithely 4200 Semiconductor Characterization System in glove-box filled Nitrogen.

\subsection{UV-Vis absorption}

Spectra were acquired on a Cary 5000 (Varian) instrument. Different P3HT solution was filled in a $0.5 \mathrm{~mm}$-thick quartz cuvette.

\subsection{Viscosity measurement}

Rheology measurements were carried out on an Anton Paar MCR-301 rheometer with a Peltier temperature control system and a cone-and plate geometry with $25 \mathrm{~mm}$ diameter plates and a $1^{\circ}$ cone angle. Viscosity measurements were carried out at a constant shear rate of $\left(100 \mathrm{~s}^{-1}\right)$, with a measuring time of $15 \mathrm{~s}$ per point. Before measurements on the polymer solutions, the viscosities of various solvents (water, toluene, etc.) were measured at $25{ }^{\circ} \mathrm{C}$, and found to agree with literature values to within $2 \%$.

\subsection{Transmission electron microscopy}

TEM experiments were carried out on a FEI Tecnai 12 operated with $120 \mathrm{kV}$ accelerating voltage. Thin film samples for TEM were prepared by floating the thin film in DI water and picking it up using a 300 mesh copper grid. The surface analysis for the samples was carried out by AFM (Agilent 5400 SPM).

\subsection{Gel permeation chromatography}

Molecular weight and molecular weight distribution of polymers was determined from SEC using an Agilent liquid chromatography system fitted with refractive index (RID) and UV-Vis detectors, using two identical PL gel columns ( $5 \mu \mathrm{m}$, MIXED-C) in connected series with THF as the mobile phase $\left(1 \mathrm{ml} \mathrm{min}{ }^{-1}\right)$. The column and flow path were temperature controlled at $25{ }^{\circ} \mathrm{C}$. Calibration was performed relative to linear PS. Data Analysis was performed using the Astra software (Wyatt Technology Corporation, Santa Barbara, USA). The results are shown in Fig. S1 (ESI $\dagger$ ).

\section{Results and discussion}

\subsection{Viscosity and entanglements in the solution state}

We begin this work by evaluating the specific viscosity of six different molecular weights of P3HT (22, 32, 68, 100, 120 and $180 \mathrm{kDa}$ ) in four key solvents of increasing solubilising capability at room temperature: toluene (Tol), chloroform $\left(\mathrm{CHCl}_{3}\right)$, chlorobenzene (CB) and dichlorobenzene (oDCB). The Hansen solubility parameters of those four solvents ${ }^{23}$ and $\mathrm{P}_{3} \mathrm{HT}^{24}$ as well as other relevant parameters are summarized in Table 1.

Table 1 The Hansen solubility parameters of the four solvents and P3HT at room temperature as well as other parameters

\begin{tabular}{|c|c|c|c|c|c|}
\hline & $\mathrm{CHCl}_{3}$ & Tol & $\mathrm{CB}$ & oDCB & P3HT \\
\hline$T_{\mathrm{b}}\left({ }^{\circ} \mathrm{C}\right)$ & 61 & 111 & 132 & 180 & - \\
\hline$\delta_{\mathrm{D}}\left(\mathrm{cal} \mathrm{cm}^{-3}\right)^{1 / 2}$ & 8.70 & 8.8 & 9.29 & 9.39 & 9.02 \\
\hline$\delta_{\mathrm{P}}\left(\mathrm{cal} \mathrm{cm}^{-3}\right)^{1 / 2}$ & 1.52 & 0.68 & 2.10 & 3.08 & 2.58 \\
\hline$\delta_{\mathrm{H}}\left(\mathrm{cal} \mathrm{cm}^{-3}\right)^{1 / 2}$ & 2.79 & 0.98 & 0.98 & 1.61 & 2.58 \\
\hline$\delta_{\mathrm{T}}\left(\mathrm{cal} \mathrm{cm}^{-3}\right)^{1 / 2}$ & 9.29 & 8.88 & 9.57 & 10.01 & 9.75 \\
\hline$\left|\Delta \delta_{\mathrm{T}}\right|=\left|\delta_{\mathrm{P} 3 \mathrm{HT}}-\delta_{\text {solvent }}\right|$ & 0.46 & 0.87 & 0.18 & 0.26 & - \\
\hline Dipole moment (D) & 1.15 & 0.36 & 1.54 & 2.14 & - \\
\hline
\end{tabular}

Comparing the total Hansen solubility parameters of P3HT with that of solvents at room temperature reveals Tol to be the poorest solvent, followed by $\mathrm{CHCl}_{3}$, while $\mathrm{CB}$ and oDCB are much better solvents for P3HT. Based on this, Tol and $\mathrm{CHCl}_{3}$ are most likely to behave as a theta solvent at room temperature, in agreement with previous observations. ${ }^{18,19}$ We have calculated the specific viscosities from the measured viscosities of the solvents (Table S1, ESI $\dagger$ ) and the solutions (Tables S2 and S3, ESI $\dagger)$. The specific viscosity $\eta_{\mathrm{sp}}$ is defined as $\eta_{\mathrm{sp}}=\left(\eta-\eta_{\mathrm{s}}\right) / \eta_{\mathrm{s}}$ with $\eta$ being the measured viscosity of the polymer solution and $\eta_{\mathrm{s}}$ the viscosity of the neat solvent. ${ }^{13}$ In Tol, the specific viscosity increases by more than an order of magnitude with the increase of $M_{\mathrm{w}}$ at a constant concentration of $5 \mathrm{mg} \mathrm{ml}^{-1}$, as seen in Fig. 1a. This indicates the presence of much stronger interactions and entanglements among the polymer chains at $M_{\mathrm{w}}$ of $100 \mathrm{kDa}$ and above, with a small effect also observed at $68 \mathrm{kDa}$. Disentangling the polymer solution via ultrasonication we observe a decrease of the specific viscosity in the high $M_{\mathrm{w}}$ cases, e.g., from 23.5 to 16.4 for the highest $M_{\mathrm{w}}$ case, whereas the lowest $M_{\mathrm{w}}$ shows no effect. A similar trend is observed for P3HT in the good solvent CB at room temperature with higher concentration, which requires increasing the concentration fivefold to $25 \mathrm{mg} \mathrm{ml}^{-1}$. Again, disentangling the solution by sonication reduces the specific viscosity of the highest $M_{\mathrm{w}}$ case sharply from close to 40 down to 13.78 while it remained unchanged for the low $M_{\mathrm{w}}$ case. In both instances we demonstrate that higher $M_{\mathrm{w}}$ P3HT can be easily driven to a highly entangled state if the concentration is adjusted with respect to the solvent and the solubility of the polymer at a given temperature. Clearly, the solvent affects how the polymer chains interact and whether entanglements are present. In one of the most widely available "high $M_{\mathrm{w}}$ " P3HT currently commercially available on the market, $68 \mathrm{kDa}$, which is above the critical $M_{\mathrm{w}}\left(M_{\mathrm{wc}} \sim 35 \mathrm{kDa}^{13}\right)$, we find the specific viscosities to be $0.42\left(\mathrm{CHCl}_{3}\right), 0.51$ (Tol), $0.39(\mathrm{CB})$, and 0.37 (oDCB) after dissolving this polymer in the four different solvents at room temperature at a concentration of $5 \mathrm{mg} \mathrm{ml}^{-1}$, as shown in Fig. 1b. The specific viscosity is slightly higher in Tol and $\mathrm{CHCl}_{3}$ where we see higher specific viscosity than in comparatively good solvents. We confirm that this is due to entanglement of polymer chains in Tol and $\mathrm{CHCl}_{3}$. This will be further discussed in Section 4.1. The disentanglement action decreases the specific viscosity in $\mathrm{CHCl}_{3}$ and in Tol, but no significant changes are seen in the other two solvents. This strongly suggests that solvophobic forces in both $\mathrm{CHCl}_{3}$ and Tol are stronger than in CB and oDCB, resulting in a significant entanglement density within polymer chains in those marginal solvents. In the solvated state, the polymer chains tend to choose the extended state with large hydrodynamic volume even for high $M_{\mathrm{w}}$, whereas in formulations where solvophobic driving forces are present the polymer chains prefer adopting a more rigid configuration. ${ }^{25}$ Disentanglement provides more freedom of movement for the chains to start folding and aggregating in the presence of solvophobic driving forces which decreases the specific viscosity of the solution.

In Fig. 1c we show how the concentration affects polymer chain entanglements in solution at a fixed $M_{\mathrm{w}}$ of $68 \mathrm{kDa}$ for the different solvents investigated here in terms of specific viscosity. As expected, the specific viscosity increases with the increasing 
a

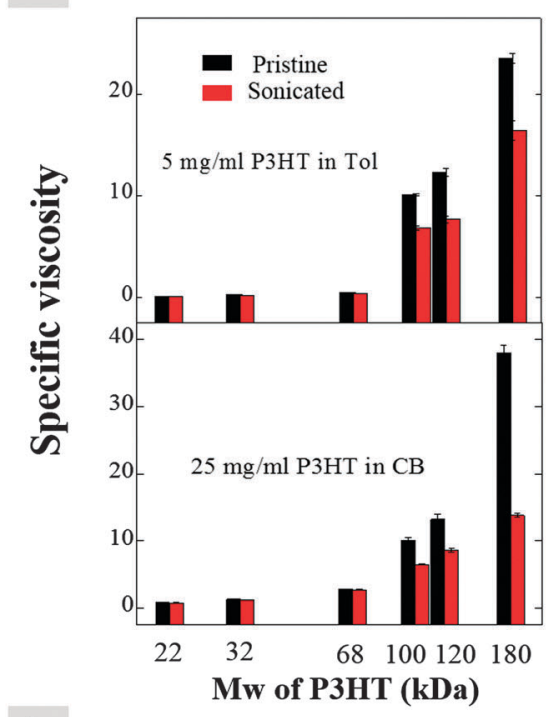

b

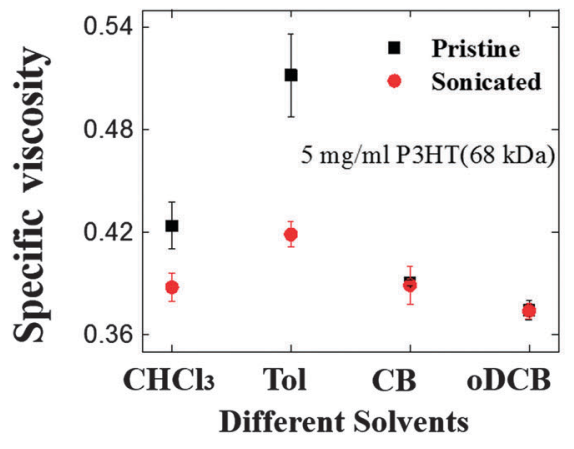

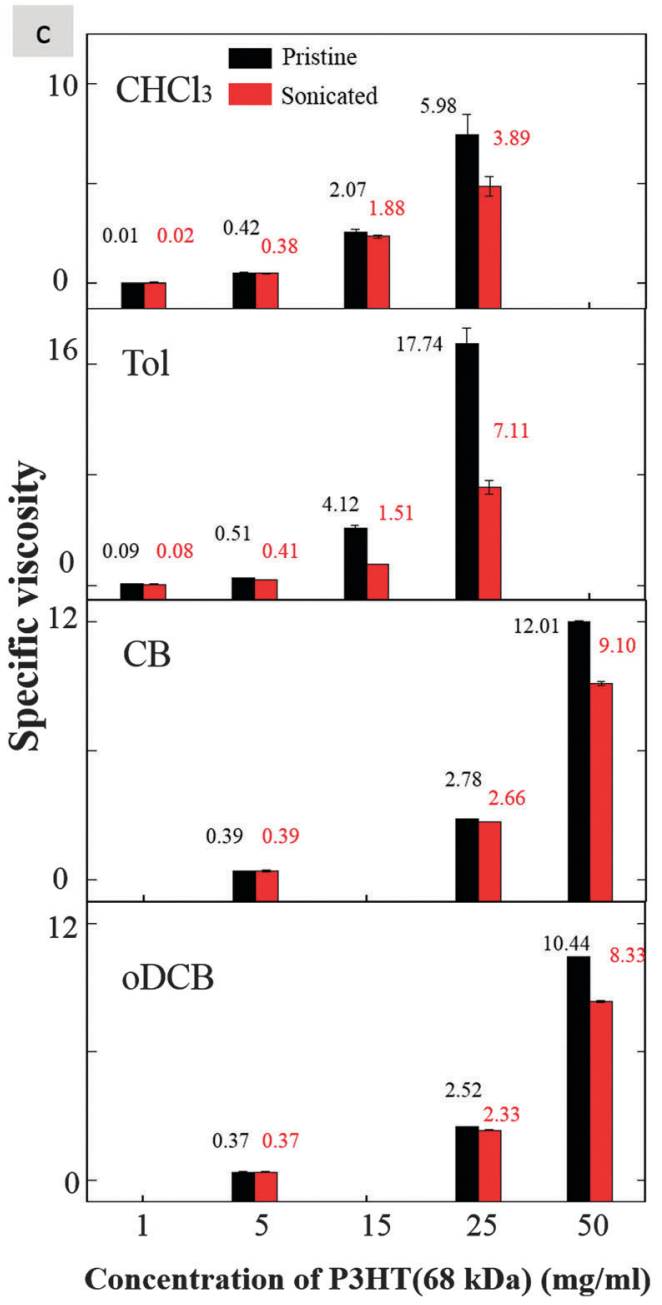

Fig. 1 Specific viscosity of room temperature P3HT solutions from different solvents and $M_{\mathrm{w}}$ before and after disentanglement (via ultrasonication). The specific viscosity of P3HT solutions with respect to $M_{w}$ both before and after disentanglement in (a) Tol (5 mg ml ${ }^{-1}$ ) (up) and CB (25 mg ml $\mathrm{m}^{-1}$ ) (down). (b) The specific viscosity of P3HT solutions $(68 \mathrm{kDa})$ with respect to solution concentration in different solvents $\left(\mathrm{CHCl}_{3}, \mathrm{Tol}_{1} \mathrm{CB}\right.$ and oDCB) before and after disentanglement. (c) The specific viscosity of $\mathrm{P} 3 \mathrm{HT}(68 \mathrm{kDa})$ solutions before and after ultrasonication from $\mathrm{CHCl}_{3}, \mathrm{Tol} \mathrm{CB}$ and $\mathrm{ODCB}$ at a concentration of $5 \mathrm{mg} \mathrm{ml}^{-1}$.

concentration of $\mathrm{P} 3 \mathrm{HT}$ in the solution, increasing from 0.09 to 17.74 for Tol from $1 \mathrm{mg} \mathrm{ml}^{-1}$ to $25 \mathrm{mg} \mathrm{ml}^{-1}$, the approximate limit for this solvent at room temperature. A similar trend can be noticed in the good solvents (CB and oDCB), in which we can readily dissolve $\mathrm{P} 3 \mathrm{HT}$ at higher concentrations. This higher concentration is required to push the system toward stronger intermolecular interactions and entanglements, from which point we can start seeing the benefits of disentanglement at room temperature via ultrasonication of the solution. The results are thus clear: increasing the concentration of P3HT results in closer interactions between the polymer chains. As more molecules entangle and the specific viscosity increases, one enters a regime in which solvophobic effects can be exploited to decrease the viscosity by disentangling of P3HT chains and inducing aggregate formation, which reduces the local concentration of non-aggregated polymer chains. With the ability to tune the solution-state aggregation in CB and oDCB solutions, there may be an opportunity to tune the bulk heterojunction formation when P3HT is blended with an acceptor, which will be the subject of future investigations.

\subsection{Disentanglement and aggregation in the solution state}

We now investigate the solution-state aggregation of P3HT using UV-Vis absorption measurements (Fig. 2a and b) performed on the pristine and disentangled solutions of identical concentration. ${ }^{12,19}$ The solution state of P3HT absorption maximum associated with the $\pi-\pi^{*}$ intraband transition appears at $c a .450 \mathrm{~nm}$ for all the solutions according to previous investigations of regioregular head-to-tail P3HT. ${ }^{26-28}$ Two additional absorption peaks at higher wavelength (i.e. lower energy) at ca. $560 \mathrm{~nm}$ and $615 \mathrm{~nm}$, are associated to 0-1 (intrachain) and 0-0 (interchain) transitions, respectively. ${ }^{29,30}$ Here, we report only the spectral range from 520 to $700 \mathrm{~nm}$, due to limitations associated to performing UV-Vis absorption measurements on high concentration solutions. Nevertheless, this range is sufficient as we can clearly see the intrachain and interchain absorption peaks, as seen in Fig. 2. 


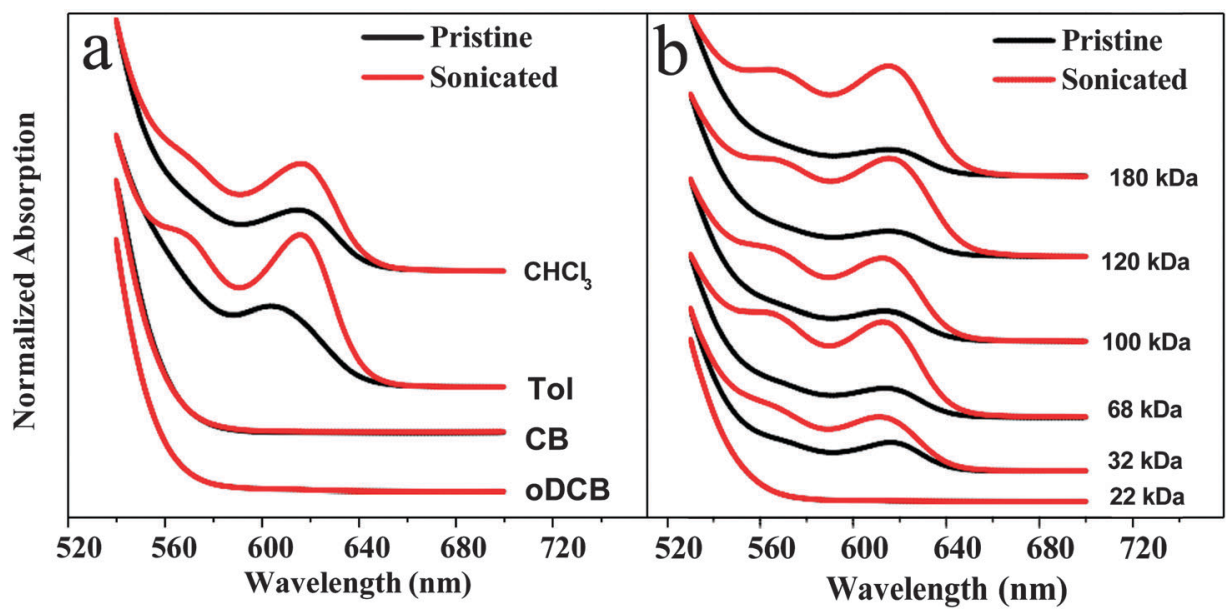

Fig. 2 Normalized (at $\lambda=530 \mathrm{~nm}$ ) UV-Vis absorption spectra of at room temperature P3HT solutions before and after disentanglement (via ultrasonication) in different solvents and using different $M_{\mathrm{w}}$. (a) Normalized UV-Vis absorption spectra of P3HT (68 kDa) solutions before and after disentanglement in $\mathrm{CHCl}_{3}, \mathrm{Tol}, \mathrm{CB}$ and oDCB. (b) Normalized UV-Vis absorption spectra of P3HT in Tol before and after disentanglement for different $M_{w}$ $(22,32,68,100,120$, and $180 \mathrm{kDa})$. All experiments performed at a concentration of $5 \mathrm{mg} \mathrm{ml}^{-1}$.

As expected, in well-dissolved solutions $\left[5 \mathrm{mg} \mathrm{ml}^{-1}\right.$ of $68 \mathrm{kDa}$ P3HT in CB or oDCB (Fig. 2a); $5 \mathrm{mg} \mathrm{ml}^{-1}$ of $22 \mathrm{kDa}$ P3HT in Tol (Fig. 2b)] we find no evidence of vibronic signatures at 560 or $615 \mathrm{~nm}$ and ultrasonicating these solutions yields no changes in their photophysical response. However, in all cases where the pristine solution shows evidence of photophysical aggregation of P3HT (Fig. 2a: $\mathrm{CHCl}_{3}$ and Tol; Fig. 2b: 32-180 kDa) we show that disentanglement induces a significant increase of the absorption in the solution-state, indicating that disentanglement enhances photophysical aggregation and effectively results in increased local order in terms of intermolecular and intramolecular interactions. These observations agree with the viscosity results that were presented in Section 2.1, where high viscosity in the pristine solution indicated the polymer chains interact more closely in these marginal/poor solubility conditions. UV-Vis absorption and viscosity measurements indicate the polymer forms highly entangled macromolecules in solution, which probably include coils, aggregates, as well as micro-crystallites. ${ }^{19}$ Disentangling the polymer chains thus reduces the specific viscosity by providing the molecules with the mobility to form more free floating or suspended aggregates with high internal local order.

In the theta solvent Tol, we observe a doubling of the normalized intensity of interchain absorption peak from 0.12 to 0.24 at $c a .613 \mathrm{~nm}$ for $32 \mathrm{kDa} 33 \mathrm{HT}$ and more than tripling of the same from 0.13 to 0.47 for $68 \mathrm{kDa}$ P3HT. Even more notable increases are seen at higher $M_{\mathrm{w}}$, as shown in Fig. $2 \mathrm{~b}$. The intrachain absorption peak becomes prominent for higher $M_{\mathrm{w}}$ samples. The increase of both interchain and intrachain absorption peaks point to micro-crystallite formation in solution, in agreement with previous dynamic light scattering and X-ray diffraction studies. ${ }^{18,31}$ The disentanglement process increases the interchain absorption peak significantly in both $\mathrm{CHCl}_{3}$ and Tol, with the intrachain absorption increasing more prominently in Tol solutions, indicating a higher backbone planarity and chain extension and perhaps longer range order is more easily achieved in the solution state when Tol is used as a solvent rather than $\mathrm{CHCl}_{3}$. Looking closely at the absorption spectra of pristine and ultrasonicated solutions in Tol, it is clear that the position of the interchain absorption peak is redshifted by $c a$. 2-3 $\mathrm{nm}$ with increasing $M_{\mathrm{w}}$, which may be due to the extended conjugation length of the polymer backbone. ${ }^{18}$ This is supported by TEM measurements of the lamellar thickness of P3HT fibrils in the solid state which is found to increase after ultrasonication, as shown in Fig. S2 (ESI $\dagger$ ). These observations support the notion that P3HT forms micro-crystallites in Tol-based solutions while aggregates exhibiting more local order are formed in the other solvents explored herein within the processing window covered in this study.

\subsection{Local order in solid state P3HT thin films}

Similar UV-Vis absorption measurements can be performed on thin films in order to correlate the solution state aggregation to the local order in the solid state. A recent model by Spano (Fig. S3, $\mathrm{ESI} \dagger$ ) has enabled the quantitative analysis of regioregular P3HT absorption spectra in relation to the morphology based on the thin film absorption spectra. ${ }^{32,33}$ The film absorption spectrum is composed of two parts, a crystalline part due to interacting chains and monomers and an amorphous contribution from disordered chains. Thin film absorption spectra of different solvents (Fig. S4) and different $M_{\mathrm{w}}$ (Fig. S5) are shown in the ESI. $\dagger$ The following eqn (1) can be used to estimate the absorption contributions of photophysical aggregates due to local order from the amorphous P3HT contributions. ${ }^{34,35}$

$$
\begin{aligned}
A(E) \propto & \sum_{m=0}\left(\frac{S^{m}}{m !}\right) \times\left(1-\frac{W \mathrm{e}^{-S}}{2 E_{\mathrm{p}}} \sum_{n \neq m} \frac{S^{n}}{n ! n-(m)}\right)^{2} \\
& \times \exp \left(\frac{\left(E-E_{0-0}-m E_{\mathrm{p}}-\frac{1}{2} W S^{m} \mathrm{e}^{-S}\right)^{2}}{2 \sigma^{2}}\right)
\end{aligned}
$$

Here, $A$ is the absorbance with respect to photon energy $(E)$, $W$ is the free exciton bandwidth of the intrachain aggregates, and $S$ is the Huang-Rhys factor with a fixed value of 1.0 taken 

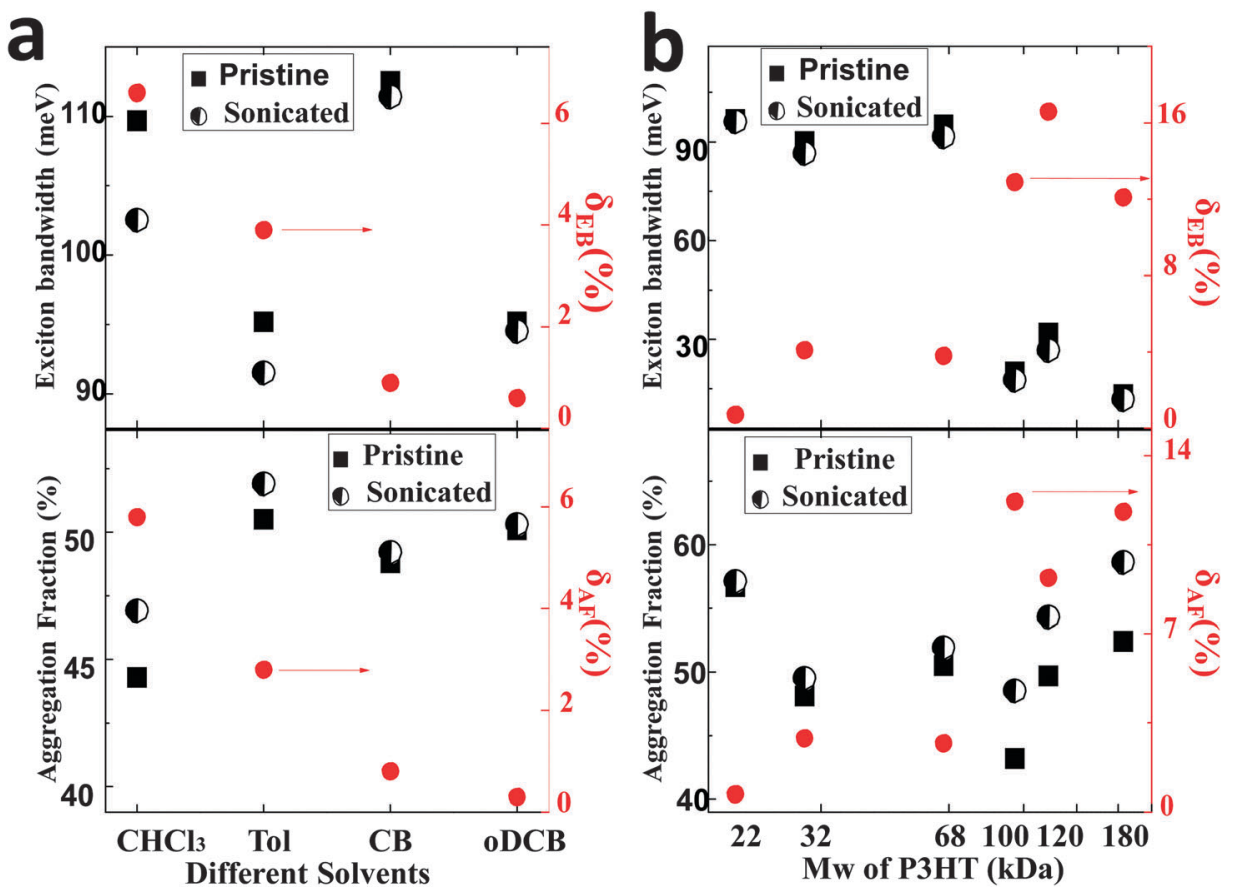

Fig. 3 The evolutions of exciton bandwidth $W$, fraction of photophysical aggregates (AF) in P3HT thin films and the changes in exciton bandwidth $\left(\delta_{\mathrm{EB}}=\left(W-W_{0}\right) / W_{0}\right)$ as well as $\mathrm{AF}\left(\delta_{\mathrm{AF}}=\left(\mathrm{AF}-\mathrm{AF}_{0}\right) / \mathrm{AF}_{0}\right)$ as a result of disentanglement (a) in different solvents at $M_{\mathrm{W}}=68 \mathrm{kDa}$ and $(\mathrm{b})$ with respect to $M_{\mathrm{W}}$ in Tol at a fixed concentration of $5 \mathrm{mg} \mathrm{ml}^{-1}$.

from a Franck-Condon fit. ${ }^{36}$ The integers $m$ and $n$ are differing vibrational levels, $E_{0-0}$ is defined as the $0-0$ transition energy, $E_{\mathrm{p}}$ is the intermolecular vibrational energy taken as $0.18 \mathrm{eV},{ }^{37}$ and $\sigma$ is the Gaussian linewidth. $W$ is inversely proportional to the width of the cofacially packed chain segments in P3HT aggregates and is therefore indicative of backbone planarity or reduced torsion.

Following ultrasonication of the solution of $68 \mathrm{kDa}$ P3HT at $5 \mathrm{mg} \mathrm{ml}{ }^{-1}, W$ decreased from 110 to $102 \mathrm{meV}$ in $\mathrm{CHCl}_{3}$ and from 95 to $90 \mathrm{meV}$ in Tol, indicating increased intrachain exciton coupling, but did not change in CB and oDCB, as shown in Fig. 3a. The fraction of photophysical aggregates also increased after ultrasonication in $\mathrm{CHCl}_{3}$ and Tol, increasing from $44.3 \%$ to $46.9 \%$ and from $50.1 \%$ to $52.0 \%$, respectively. These trends agree with the solution state analysis previously performed and indicate that the backbone planarity and increased local order in the solid state can be controlled in the solution state by disentangling the solution in formulation conditions of marginal solubility. The reduction in $W$ can be understood as an extension of the conjugation length within polymer chains, in good agreement with the red-shift of the intramolecular absorption peak of P3HT in the same solutions and with the increased lamellar thickness in P3HT fibrils (Fig. S2, ESI $\dagger$ ). A similar investigation performed at different $M_{\mathrm{w}}$ of P3HT is shown in Fig. 3b. A significant decrease of the exciton bandwidth is observed for pristine solutions prepared for P3HT with high $M_{\mathrm{w}}$ samples $^{38}\left(M_{\mathrm{w}} \geq 100 \mathrm{kDa}\right)$. Here, ultrasonication reduces the exciton bandwidth even further by $12-16 \%$, indicating that the polymer chains were highly entangled in solution. The fraction of aggregates also increases substantially, e.g., from $52.4 \%$ to $58.6 \%$ for $M_{\mathrm{w}}=180 \mathrm{kDa}$, a change of about $12 \%$.

\subsection{Thin film microstructure and morphology}

In Fig. 4a we show TEM micrographs of P3HT thin films (68 kDa; $5 \mathrm{mg} \mathrm{ml}^{-1}$ ) spin-cast from pristine and ultrasonicated Tol and CB solutions. The film prepared from Tol exhibits a fibril structure, whereas the CB films show no distinctive morphological or microstructural features in the micrograph. The fibrils are believed to be seeded in the solution, as discussed in Section 2.2 where we observed appearance of solution state aggregates with good intermolecular interactions and backbone planarity in Tol but not in CB. TEM and AFM micrographs of P3HT ( $68 \mathrm{kDa}$ ) thin films prepared from $\mathrm{CHCl}_{3}$ and oDCB are shown in Fig. S6 (ESI $\dagger$ ). In the case of $\mathrm{CHCl}_{3}$, we observe nodule formation after ultrasonicating the solution, in agreement with previous reports..$^{12,18,19}$ Selected area electron diffraction (ED) measurements were performed to investigate the crystallinity in the $\pi$-stacking direction. The $\pi$-stacking ring (020) is clear for all the samples, indicating the presence of long range order irrespective of the presence or absence of fibrils or nodules in the film. We have plotted in Fig. S7 (ESI $\dagger$ ) the normalized integrated ED spectra obtained for films cast from all four solvents for pristine and ultrasonicated solutions. The ED results reveal increased (020) intensity in the $\mathrm{CHCl}_{3}$ and Tol cases after ultrasonication, but no apparent change in either CB or oDCB, again highlighting that disentanglement and aggregation in the solution in the presence of solvophobic forces leads to greater long range order in the $\pi$-stacking direction in thin films.

AFM micrographs of P3HT films obtained from pristine and ultrasonicated solutions in Tol for $M_{\mathrm{w}}=22$ and $180 \mathrm{kDa}$ are compared in Fig. 4b. We observe no effect on the surface morphologies in the low $M_{\mathrm{w}}$ case. This is consistent with classical polymer 

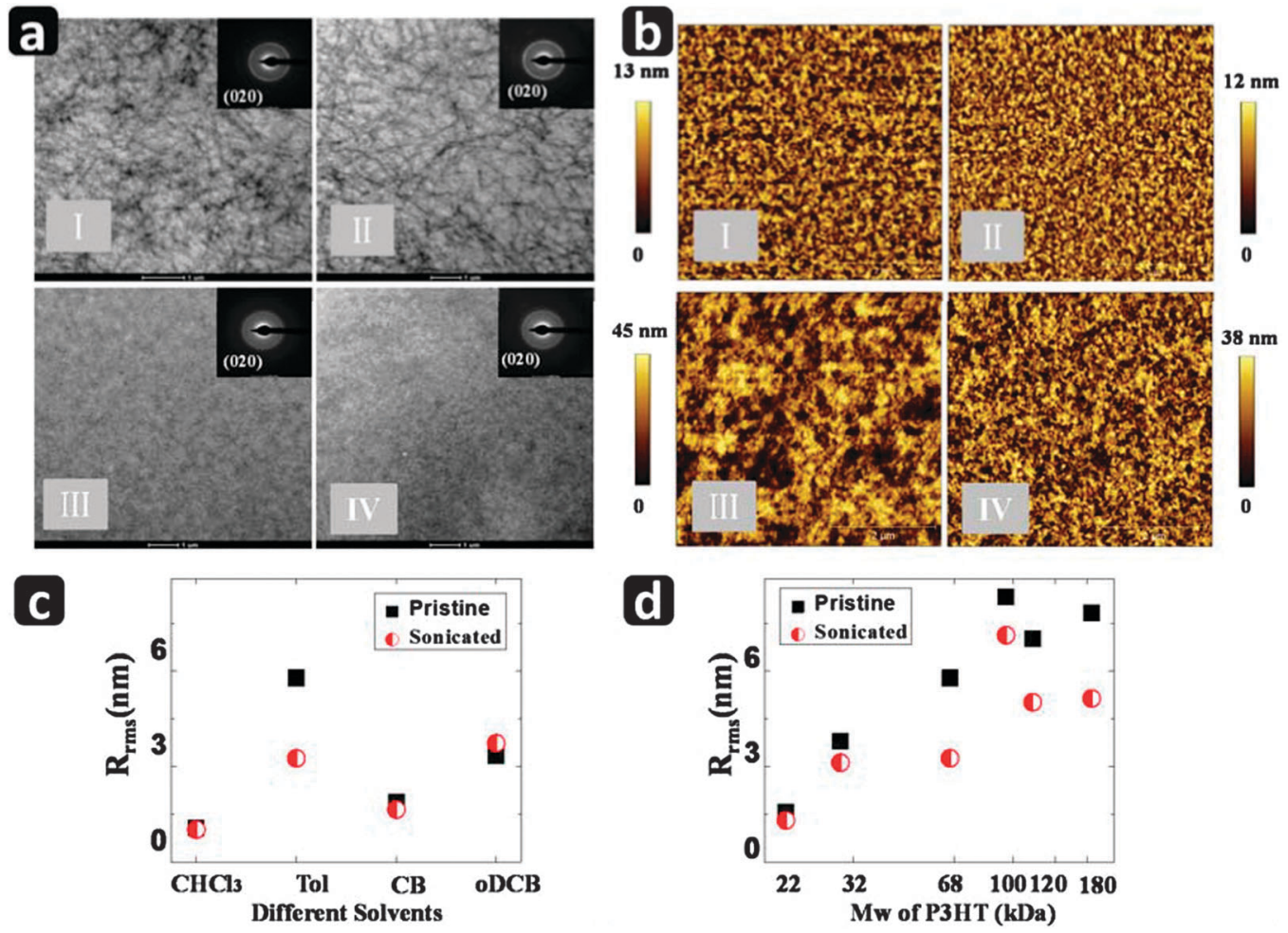

Fig. 4 (a) Bright-field TEM image of P3HT (68 kDa) thin films prepared from different solvents: Tol (I) pristine, (II) disentangled, CB (III) pristine, (IV) disentangled. The insets show electron diffraction (ED) images. (b) AFM micrographs of surface topography of P3HT thin films prepared from different $M_{\mathrm{w}}$ of P3HT in Tol: $22 \mathrm{kDa}$ (I) pristine and (II) disentangled; $180 \mathrm{kDa}$ (III) pristine and (IV) disentangled. (c) Surface roughness (root mean squared) of P3HT $(68 \mathrm{kDa})$ thin films prepared from different solvents before and after disentanglement. (d) Surface roughness of P3HT thin films from Tol before and after disentanglement for different $M_{\mathrm{w}}(22 \mathrm{kDa}, 32 \mathrm{kDa}, 68 \mathrm{kDa}$ and $100 \mathrm{kDa}, 120 \mathrm{kDa}$ and $180 \mathrm{kDa})$. All solution concentrations are $5 \mathrm{mg} \mathrm{ml}{ }^{-1}$.

science, according to which polymers with a low molecular weight form isolated, extended-chain crystals, which result in a polycrystalline one-phase morphology, ${ }^{38}$ in agreement with the micrographs. In contrast, longer chain P3HT forms a two-phase morphology consisting of alternating crystalline lamellae and disordered regions, which are interconnected by individual macromolecular tie molecules bridging multiple ordered domains. ${ }^{39}$ The disentanglement of long polymer chains not only facilitates the molecular packing which results in the extended conjugation along the polymer backbone and in increased photophysical aggregate fraction, but also affects the morphology of the thin films. For instance, while the lamellar thickness of the fibrils increases after disentanglement for the $68 \mathrm{kDa}$ samples, feature sizes decrease sharply for the higher $M_{\mathrm{w}}$ P3HT after disentanglement, as shown in Fig. S8 (ESI $\dagger$ ). This is believed to be due to the formation of smaller aggregates with improved diffusivity and dispersion of the aggregated precursors in solution. ${ }^{19}$ In Fig. $4 \mathrm{c}$ and $\mathrm{d}$ we plot the surface roughness of the thin films processed from different solvents and different $M_{\mathrm{w}}$ P3HT, respectively, from pristine and disentangled solutions. For thin film samples prepared from marginal and highly entangled solutions (e.g., Tol and/or high $M_{\mathrm{w}}$ ) display significant decreases of the surface roughness across the board after ultrasonication.

\subsection{Carrier transport}

Bottom-gate, bottom-contact OTFTs were fabricated by spincoating the pristine and ultrasonicated solutions of different $M_{\mathrm{w}}$ in different solvents on a substrate pre-coated with source and drain electrodes. The hole mobility of OTFT devices from different solvents and different $M_{\mathrm{w}}$ are shown in Fig. 5a and b while transfer characteristics are summarized in Fig. S9 (ESI $\dagger$ ). We observe significant improvements in the hole mobility after ultrasonication of the $\mathrm{CHCl}_{3}(\sim 2 \times)$ and $\mathrm{Tol}(\sim 3 \times)$ solutions (i.e., from 0.0030 to $0.0063 \mathrm{~cm}^{2} \mathrm{~V}^{-1} \mathrm{~s}^{-1}$ in $\mathrm{CHCl}_{3}$ and from 0.0050 to $0.013 \mathrm{~cm}^{2} \mathrm{~V}^{-1} \mathrm{~s}^{-1}$ in Tol), but no change to the samples prepared from good solvents such as CB and oDCB in these low concentration conditions. We also observe a significant improvement of mobility with ultrasonication of P3HT solutions of increasing $M_{\mathrm{w}}$ at $5 \mathrm{mg} \mathrm{m}^{-1}$, as seen in Fig. $5 \mathrm{~b}$. The carrier mobility of high $M_{\mathrm{w}}$ P3HT ( $>100 \mathrm{kDa}$ ) can be increased up to six-fold from $\sim 0.01$ up to $\sim 0.06 \mathrm{~cm}^{2} \mathrm{~V}^{-1} \mathrm{~s}^{-1}$, highlighting the significant benefits of this disentanglement approach upon the transport properties of highly entangled and marginally solvated high $M_{\mathrm{w}}$ conjugated polymers.

\section{Discussion}

\subsection{Entanglements and polymer chain behaviour in the solution state}

The presence of polymer chain entanglements depends strongly upon molecular weight, because of the latter's effect on chain contour length and tortuosity. ${ }^{40}$ Based on the tube model as 

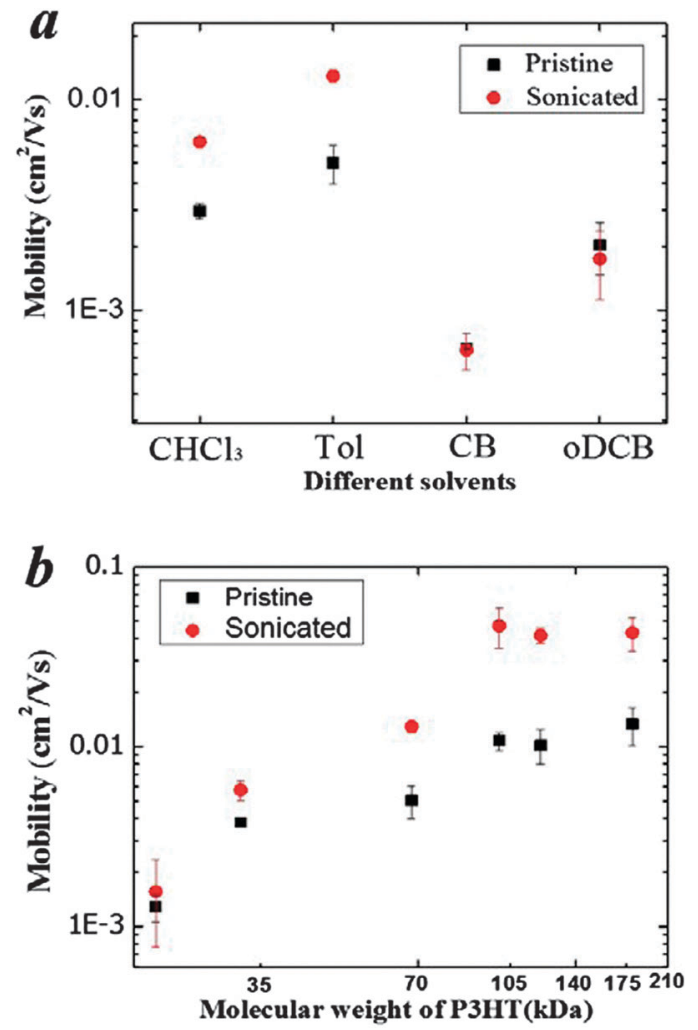

Fig. 5 Field effect hole mobility measured in the BC-BG OTFT configuration. (a) Carrier mobility for $68 \mathrm{kDa}$ P3HT prepared from different solvents before and after the disentanglement process. (b) Carrier mobility of OTFTs different molecular weight from Tol.

shown in Fig. 6a, the persistence length ${ }^{10,11}\left(l_{\mathrm{p}}\right)$ of P3HT was reportedly around $2.4 \mathrm{~nm}^{41}$ although this is believed to differ depending on the solvent environment. ${ }^{42}$ The average molecular chain contour length, $L_{\text {chain }}$, is expressed as $L_{\text {chain }}=l_{0} M_{\mathrm{w}} /\left(M_{0} B\right)$, where $l_{0}=3.8 \AA$, is the theoretical value of the repeat unit of 3hexylthiophene, ${ }^{43} M_{0}=166.3 \mathrm{~g} \mathrm{~mol}^{-1}$, and $B$ is a coefficient whose value is 2.0 for conjugated molecules. ${ }^{44}$ For a low $M_{\mathrm{w}}$ polymer [below the critical $M_{\mathrm{w}}\left(M_{\mathrm{wc}}\right)$ of $35 \mathrm{kDa}$ for $\mathrm{P}_{3} \mathrm{HT}^{13}$ ], the persistence length plays an important role and results in a higher rigidity of the backbone, which becomes rod-like, as illustrated in Fig. 6a. For a high $M_{\mathrm{w}}$ polymer $\left(M_{\mathrm{w}}>M_{\mathrm{wc}}\right)$, the effect of the persistence length is almost negligible due to the large contour length of the polymer chain $\left(L_{\text {chain }} \gg l_{\mathrm{p}}\right)$ and the polymer shows greater flexibility with chain folding and distortion occurring. ${ }^{45}$ At the same time, high $M_{\mathrm{w}}$ polymers are less soluble due to a reduced entropy of mixing resulting in greater solvophobic forces with increasing $M_{\mathrm{w}}$. This decreases the effective hydrodynamic volume of polymer molecules and forces the polymer chains to interact more closely with a greater chance of entanglement. It is therefore not a surprise that high $M_{\mathrm{W}}$ P3HT should suffer from more entanglements, ${ }^{46}$ and consequently exhibit a higher specific viscosity as was shown in Fig. 1. The intensified solvophobic forces promote polymer coiling and intermolecular interactions between polymer chains, which leads to the formation of precursor species in the solution, as detected by UV-Vis absorption (Fig. 2).
For a given polymer with $M_{\mathrm{w}}>$ critical $M_{\mathrm{w}}\left(M_{\mathrm{wc}}\right)$, the effective hydrodynamic volume is largest at infinite dilution, where the polymer chains are completely isolated from other solute molecules. The polymer chain's extension is also at a maximum depending on the extent of interactions with the solvent, ${ }^{47}$ as schematically illustrated in Fig. S10 (ESI $\dagger$ ). There are two factors influencing the effective hydrodynamic volume directly: the concentration and the nature of the solvent. The effective hydrodynamic volume of the polymer molecules decrease rapidly with increase in the concentration of the solution due to the crowding of polymer molecules, and the related entanglements, as illustrated in Fig. 6c. This explanation is supported by the viscosity measurements presented in Fig. 1b according to which the specific viscosity increases sharply with increasing concentration for all solvents investigated. On the other hand, the extension of a polymer chain in a given solution with a given concentration is a function of the polymer-solvent interactions. For instance, in a good solvent the strong interactions between the polymer and the solvent lead the polymer to be more extended and to have a greater hydrodynamic volume as illustrated in Fig. $6 \mathrm{~b}$. By contrast, in a poor solvent, where the environment is less favourable to the extension of polymer chains, the polymer effectively shrinks by coiling or aggregating leading to a much smaller effective hydrodynamic volume (Fig. 6c). In the presence of long neighbouring chains, the coiling and aggregation processes raise the likelihood that significant entanglements occur which increase viscosity (Fig. 1a) and kinetically inhibit further self-assembly and folding processes responsible for aggregation. For this reason, the specific viscosity is believed to be lower in good solvents (CB and oDCB) than in poor solvents (Tol and $\mathrm{CHCl}_{3}$ ) for otherwise identical conditions.

In the cases where $M_{\mathrm{w}}<M_{\mathrm{wc}}$ (Fig. 6a) and $M_{\mathrm{w}}>M_{\mathrm{wc}}$ in a good solvent (Fig. 6b), ultrasonication does not appear to change the solution state, as shown in Fig. $6 \mathrm{a}^{\prime}$ and $\mathrm{b}^{\prime}$, respectively, in agreement with the specific viscosity and UV-Vis absorption measurements. However, when $M_{\mathrm{w}}>M_{\mathrm{wc}}$ in the presence of poor solubility (Fig. 6c), ultrasonication leads to significant changes with more pre-aggregation forming and fewer entanglements as compared with the pristine state of the solution. According to the tube model, the cube diameter $L_{\perp}$ in this system is smaller due to strong constraints from the surrounding polymer chains which also results in controlled conformation and Brownian movement. ${ }^{11}$ Ultrasonication intensifies the movement of polymer chains by increasing the cube diameter $L_{\perp}$, in other words, it increases the effective hydrodynamic volume, ${ }^{47}$ resulting in an improved freedom of movement of the molecules. This not only improves the diffusion of the molecular chains along their own contour but also weakens the suppression of movement by the surrounding molecules, resulting in modified transverse, reptational and rotational diffusion behaviours. Moreover, the deflection length $l_{\mathrm{d}}$ which was described as the flexible part of the chain is related to the diameter of the cube: $l_{\mathrm{d}} \propto L_{\perp}{ }^{2 / 3} l_{\mathrm{p}}{ }^{1 / 3} \cdot{ }^{48}$ Increased cube diameter increases the ratio of the flexible chain, resulting in intensified Brownian movement which facilitates more conformational changes. As the number of polymer aggregates increases, there are fewer dissolved molecules in the 


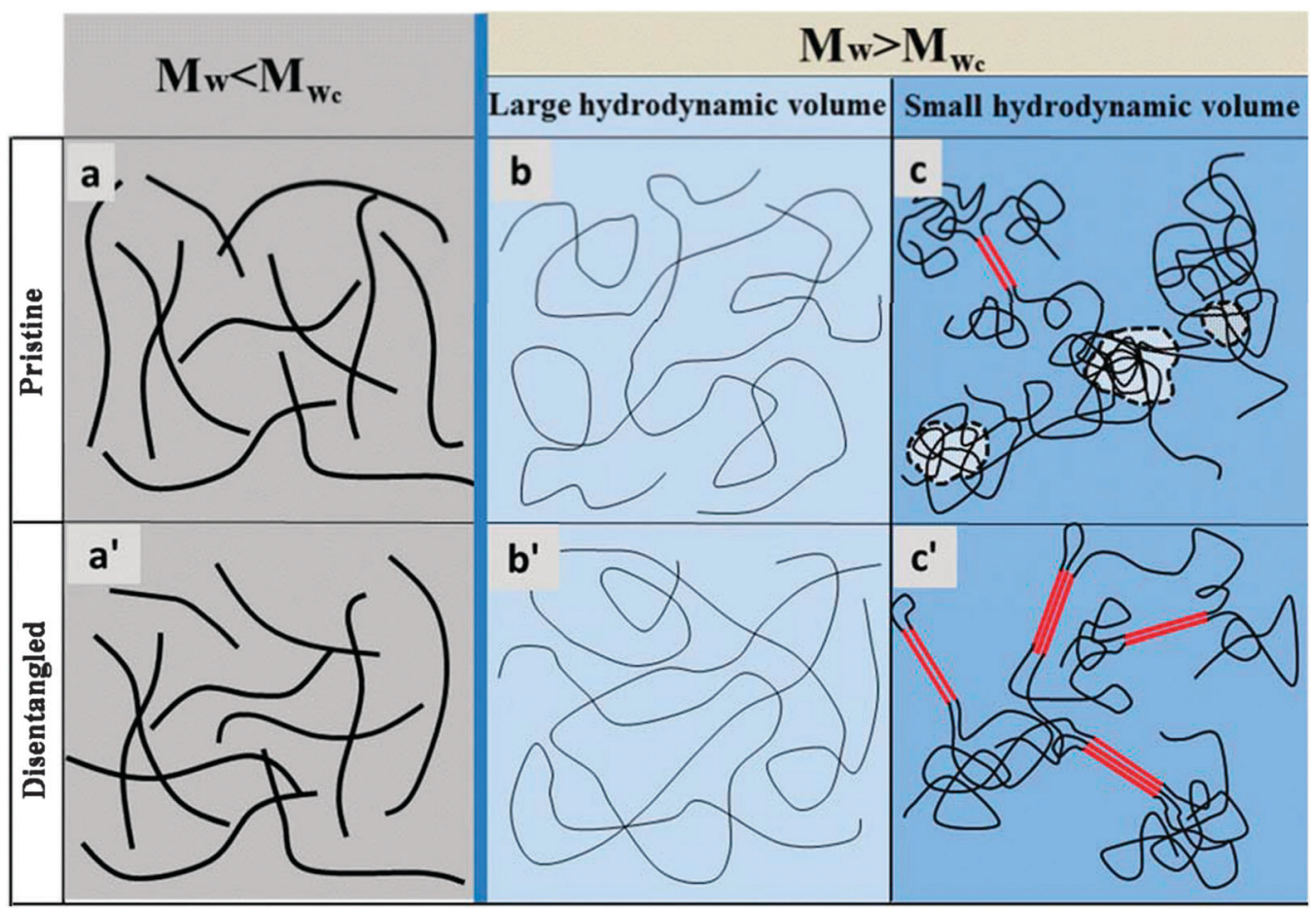

Fig. 6 Schematic model for a conjugated polymer exhibiting different molecular states in solution. (a) The low $M_{w}\left(M_{w}<M_{w c}\right)$ polymer exhibits more backbone rigidity and is in a rod-like state. ( $\left.a^{\prime}\right)$ Ultrasonication induces no change to the state of the polymer chain. (b) The solution-states of a well dissolved high $M_{w}\left(M_{w}>M_{w c}\right)$ polymer showing large hydrodynamic volume and extension of the flexible polymer chain with few interactions among the polymer molecules. ( $\left.b^{\prime}\right)$ Ultrasonication indices no significant changes to the state of polymer chain due to the large effective hydrodynamic volume and weak interactions among the molecules. (c) The solution-state of a high $M_{w}\left(M_{w}>M_{w c}\right)$ polymer in the presence of solvophobic forces exhibits small hydrodynamic volume, a tendency to aggregate but also more crowding of the flexible polymer chains, more interactions and chain entanglements with neighboring molecules, which raise the viscosity and hinder ordered aggregation in the solution. (c') Ultrasonication disentangles the polymer chains, increasing the freedom of polymer chain motion, resulting in a decrease of viscosity as well as in the formation of more ordered aggregates in the solution.

solution and thus the effective concentration of dissolved chains decreases. All of those kinetic factors therefore promote solution state aggregation, which translates to benefits in the solid state of the conjugated polymer thin film.

\subsection{Implications for electronics and photovoltaics}

The tube model predicts that $M_{\mathrm{w}}$ is a key parameter capable of tuning the properties of semi-flexible polymer chains, including the rigidity of the backbone, which strongly affects the polymer chain state in the liquid state. In the melt, the number of entanglements (N) per chain is directly proportional to $M_{\mathrm{w}},{ }^{13}$ which means that chain extension generally is inhibited. In solution, $N$ is in addition affected by the dilution/solvent concentration. In highly dilute solutions, essentially no entanglements persist. In contrast when the formulations approach the solubility limit, a relatively high disentanglement density exists. We show here that this can be beneficial to induce pre-aggregation in solution with good local and possibly even long range order. In the solid state, this results in improved local and long range orders in the $\pi$-stacking direction as well as in increased backbone planarity and lamellar thickness of the conjugated polymer chains. This ordering works effectively in high $M_{\mathrm{w}}$ polymers where tie molecules link crystalline domains. ${ }^{14}$ By contrast, transport in non-interconnected chain-extended crystals, e.g., low $M_{\mathrm{w}}$ films, is limited by domain boundaries which act as deep traps or transport barriers. ${ }^{38,49}$ These results are consistent with previous efforts made to control the state of solution in order to tune the transport properties of conjugated polymers in the solid state by the use of mixed solvents. ${ }^{50}$

Along with the main theme, we have demonstrated that solution-state aggregation can be achieved even in so-called good solvents, such as $\mathrm{CHCl}_{3}$, CB and oDCB, if one understands the solvent-concentration- $M_{\mathrm{w}}$ nexus, which pushes the formulation to exhibit greater solvophobicity. These solvents are more commonly used in organic photovoltaics, because of their ability to dissolve both donor polymers or oligomers and the soluble fullerene acceptor, thus making such concepts potentially important for organic photovoltaic applications.

A potential outcome of the work presented in this paper is its impact on helping achieve good carrier mobility and ultimately good carrier extraction and fill factor (FF) from 300-500 nm thick bulk heterojunction (BHJ) layers. Thick BHJ solar cells are under urgent demand, since they are not only more compatible to solution printing technology, a probable commercialization processing for OPVs, but also more effective in the harvesting of incident sunlight. ${ }^{51,52}$ However, increasing the thickness of the active layer not only elongates the distance for charge-carriers to travel to reach the device electrode, but also decreases the built-in electric field. ${ }^{53}$ Both of those would increase the time needed to extract the charge-carriers in the device, 
resulting in higher probability of recombination, consequently low FF and decreased PCE. ${ }^{5,55}$ To overcome these problems, higher hole mobility conjugated polymers have been successfully utilized to make thick (300 nm) OPVs with high $\mathrm{FF},{ }^{52,53}$ and along the way showing that FF is quite sensitive to the carrier mobility. It is clear that the ability to disentangle highly concentrated solutions may have several important benefits: (i) reduced specific viscosity to improve processability and possibly increase the maximum polymer loading in the solution and thus the thickness of the active layer, (ii) pre-aggregation of highly ordered and uniformly sized microcrystallites or particles, (iii) aggregates acting as seeds for more facile and reliable subsequent solution-tosolid phase transformation and growth of high quality donor domains which can achieve fast hole transport through the thick bulk heterojunction and (iv) even facilitation of the phase separation between the donor and acceptor which can easily vitrify ascast blends ${ }^{56}$ without the need for harmful solvent additives. This of course will have to happen without hindering the formation of the increasingly important mixed phase. ${ }^{57-59}$

\section{Conclusion}

We have investigated in detail the factors influencing the solution state of conjugated polymers (here exemplified by $\mathrm{P} 3 \mathrm{HT}$ ) in terms of the choice of solvent, solution concentration and molecular weight. We have identified using a combination of rheology and solution UV-Vis absorption that formulation regimes where solvophobic driving forces are present lead to increased entanglements, which hinder the aggregation behaviour. This provides an opportunity to influence the aggregation state of the solute by disentangling the polymer via ultrasonication of the solution, with consequences on the local and long range order and backbone planarity and lamellar thickness in the solid state. These parameters, in turn, affect the transport properties through the conjugated polymer in the solid state, as demonstrated by OFET devices.

We have discussed the implications of this study more broadly on polymer processing and on our ability to control polymer aggregation in solution and ordering in the solid state. We also suggest that these results should have significant implications on organic photovoltaics, where the combination of thick solar cells and fabrication from environmentally friendly solvents means that formulations are likely to be highly entangled and in the presence of significant solvophobic forces in conditions of solar cell manufacturing.

\section{Author contributions}

The manuscript was written through contributions of all authors. All authors have given approval to the final version of the manuscript.

\section{Acknowledgements}

This work was supported by the Office of Competitive Research Funds at the King Abdullah University of Science and Technology.
N.S., M.H. and A.A. acknowledge the Office of Competitive Research Funds for AEA3 awards. A.A. acknowledges SABIC for the Career Development SABIC Chair. This publication was based on work supported by award no. KUS-C1-018-02, made by King Abdullah University of Science and Technology (KAUST). We acknowledge Prof. Yu Han for help with acquisition of UV-Vis spectra at KAUST, and Dr. Misjudeen Raji for help with GPC experiments at KAUST.

\section{References}

1 J. Mei, Y. Diao, A. L. Appleton, L. Fang and Z. Bao, J. Am. Chem. Soc., 2013, 135, 6724.

2 M. S. AlSalhi, J. Alam, L. A. Dass and M. Raja, Int. J. Mol. Sci., 2011, 12, 2036.

3 C. Tang, X. D. Liu, F. Liu, X. L. Wang, H. Xu and W. Huang, Macromol. Chem. Phys., 2013, 214, 314.

4 A. J. Heeger, Chem. Soc. Rev., 2010, 39, 2354.

5 F. C. Krebs, Sol. Energy Mater. Sol. Cells, 2009, 93, 394.

6 C. M. Amb, M. R. Craig, U. Koldemir, J. Subbiah, K. R. Choudhury, S. A. Gevorgyan, M. Jørgensen, F. C. Krebs, F. So and J. R. Reynolds, ACS Appl. Mater. Interfaces, 2012, 4, 1847.

7 W. Yue, T. T. Larsen-Olsen, X. Hu, M. Shi, H. Chen, M. Hinge, P. Fojan, F. C. Krebs and D. Yu, J. Mater. Chem. A, 2013, 1, 1785.

8 H. Youn, T. Lee and L. J. Guo, Energy Environ. Sci., 2014, 7, 2764.

9 F. Bueche, J. Chem. Phys., 1952, 20, 1959.

10 P. G. de Gennes, Scaling Concepts in Polymer Physics, Cornell University Press, New York, 1979.

11 M. Doi, The theory of polymer dynamics, Oxford University Press, Oxford, 1986.

12 K. Zhao, L. Xue, J. Liu, X. Gao, S. Wu, Y. Han and Y. Geng, Langmuir, 2010, 26, 471.

13 F. P. V. Koch, J. Rivnay, S. Foster, C. Müller, J. M. Downing, E. Buchaca-Domingo, P. Westacott, L. Yu, M. Yuan, M. Baklar, Z. Fei, C. Luscombe, M. A. McLachlan, M. Heeney, G. Rumbles, C. Silva, A. Salleo, J. Nelson, P. Smith and N. Stingelin, Prog. Polym. Sci., 2013, 38, 1978.

14 R. J. Kline, M. D. McGehee, E. N. Kadnikova, J. Liu and J. M. J. Fréchet, Adv. Mater., 2003, 15, 1519.

15 S. Joshi, PhD thesis, Siegen University, Germany, 2008.

16 H. Wang, Y. Xu, X. Yu, R. Xing, J. Liu and Y. Han, Polymers, 2013, 5, 1272.

17 B. S. Ong, Y. Wu, P. Liu and S. Gardner, Adv. Mater., 2005, 17, 1141.

18 A. R. Aiyar, J. Il Hong, R. Nambiar, D. M. Collard and E. Reichmanis, Adv. Funct. Mater., 2011, 21, 2652.

19 K. Zhao, H. U. Khan, R. Li, Y. Su and A. Amassian, Adv. Funct. Mater., 2013, 23, 6024.

20 D. Choi, M. Chang and E. Reichmanis, Adv. Funct. Mater., 2015, 25, 920.

21 P. Westacott, J. R. Tumbleston, S. Shoaee, S. Fearn, J. H. Bannock, J. B. Gilchrist, S. Heutz, J. DeMello, 
M. Heeney, H. Ade, J. R. Durrant, D. S. McPhail and N. Stingelin, Energy Environ. Sci., 2013, 6, 2756.

22 R. S. Loewe, S. M. Khersonsky and R. D. McCullough, Adv. Mater., 1999, 11, 250.

23 F. MacHui, S. Langner, X. Zhu, S. Abbott and C. J. Brabec, Sol. Energy Mater. Sol. Cells, 2012, 100, 138.

24 F. MacHui, S. Abbott, D. Waller, M. Koppe and C. J. Brabec, Macromol. Chem. Phys., 2011, 212, 2159.

25 M. Koppe, C. J. Brabec, S. Heiml, A. Schausberger, W. Duffy, M. Heeney and I. McCulloch, Macromolecules, 2009, 42, 4661.

26 M. J. Winokur, D. Spiegel, Y. Kim, S. Hotta and A. J. Heeger, Synth. Met., 1989, 28, 419.

27 S. Hotta, S. D. D. V. Rughooputh, A. J. Heeger and F. Wudl, Macromolecules, 1987, 20, 212.

28 M. C. Gurau, D. M. Delongchamp, B. M. Vogel, E. K. Lin, D. A. Fischer, S. Sambasivan and L. J. Richter, Langmuir, 2007, 23, 834 .

29 P. Brown, D. Thomas, A. Köhler, J. Wilson, J.-S. Kim, C. Ramsdale, H. Sirringhaus and R. Friend, Phys. Rev. B: Condens. Matter Mater. Phys., 2003, 67, 1.

30 S. Rughooputh and S. Hotta, J. Polym., 1987, 25, 1071.

31 S. Samitsu, T. Shimomura, S. Heike, T. Hashizume and K. Ito, Macromolecules, 2008, 41, 8000.

32 F. C. Spano, J. Chem. Phys., 2005, 122, 234701.

33 F. C. Spano, Chem. Phys., 2006, 325, 22.

34 P. Pingel, A. Zen, R. D. Abellón, F. C. Grozema, L. D. A. Siebbeles and D. Neher, Adv. Funct. Mater., 2010, 20, 2286.

35 S. T. Turner, P. Pingel, R. Steyrleuthner, E. J. W. Crossland, S. Ludwigs and D. Neher, Adv. Funct. Mater., 2011, 21, 4640. 36 J. Clark, C. Silva, R. H. Friend and F. C. Spano, Phys. Rev. Lett., 2007, 98, 1.

37 G. Louarn, M. Trznadel, J. P. Buisson, J. Laska, A. Pron, M. Lapkowski and S. Lefrant, J. Phys. Chem., 1996, 100, 12532.

38 O. G. Reid, J. A. N. Malik, G. Latini, S. Dayal, N. Kopidakis, C. Silva, N. Stingelin and G. Rumbles, J. Polym. Sci., Part B: Polym. Phys., 2012, 50, 27.

39 G. Ungar, J. Stejny, A. Keller, I. Bidd and M. C. Whiting, Science, 1985, 229, 386.

40 W. W. Graessley and S. F. Edwards, Polymer, 1981, 22, 1329.
41 B. Grevin, P. Rannou, R. Payerne, A. Pron and J. P. Travers, J. Chem. Phys., 2003, 118, 7097.

42 J. Liu, R. S. Loewe and R. D. McCullough, Macromolecules, 1999, 32, 5777.

43 G. W. Heffner and D. S. Pearson, Macromolecules, 1991, 24, 6295.

44 S. Donald, A. M. Windle and A. H. Hanna, Liquid crystalline polymers, Cambridge University Press, 2006.

45 T. Odijk, Macromolecules, 1983, 1344, 1340.

46 S. M. Aharoni, Macromolecules, 1983, 16, 1722.

47 S. H. Maron, N. Nakajima and I. M. Krieger, J. Polym. Sci., 1959, 37, 1.

48 H. Yamakawa, J. Chem. Phys., 1973, 59, 3811.

49 R. J. Kline, M. D. Mcgehee, E. N. Kadnikova, J. Liu and M. J. Jean, Adv. Mater., 2003, 15, 1519.

50 T. Q. Nguyen, V. Doan and B. J. Schwartz, J. Chem. Phys., 1999, 110, 4068.

51 G. Dennler, M. C. Scharber and C. J. Brabec, Adv. Mater., 2009, 21, 1323.

52 Z. Chen, P. Cai, J. Chen, X. Liu, L. Zhang, L. Lan, J. Peng, Y. Ma and Y. Cao, Adv. Mater., 2014, 26, 2586.

53 W. Li, K. H. Hendriks, W. S. C. Roelofs, Y. Kim, M. M. Wienk and R. A. J. Janssen, Adv. Mater., 2013, 25, 3182.

54 Z. M. Beiley, E. T. Hoke, R. Noriega, J. Dacuña, G. F. Burkhard, J. A. Bartelt, A. Salleo, M. F. Toney and M. D. McGehee, Adv. Energy Mater., 2011, 1, 954.

55 S. Foster, F. Deledalle, A. Mitani, T. Kimura, K.-B. Kim, T. Okachi, T. Kirchartz, J. Oguma, K. Miyake, J. R. Durrant, S. Doi and J. Nelson, Adv. Energy Mater., 2014, 4, 1400311.

56 K. W. Chou, B. Yan, R. Li, E. Q. Li, K. Zhao, D. H. Anjum, S. Alvarez, R. Gassaway, A. Biocca, S. T. Thoroddsen, A. Hexemer and A. Amassian, Adv. Mater., 2013, 25, 1923.

57 T. M. Burke and M. D. McGehee, Adv. Mater., 2014, 26, 1923. 58 J. A. Bartelt, Z. M. Beiley, E. T. Hoke, W. R. Mateker, J. D. Douglas, B. A. Collins, J. R. Tumbleston, K. R. Graham, A. Amassian, H. Ade, J. M. J. Fréchet, M. F. Toney and M. D. Mcgehee, Adv. Energy Mater., 2013, 3, 364.

59 S. Sweetnam, K. R. Graham, G. O. Ngongang Ndjawa, T. Heumüller, J. A. Bartelt, T. M. Burke, W. Li, W. You, A. Amassian and M. D. McGehee, J. Am. Chem. Soc., 2014, 136, 14078. 